\title{
Contrast and conspicuity in preattentive texture perception
}

\author{
STEVEN TAYLOR and GORDON STANLEY \\ University of Melbourne, Parkville, Australia
}

\begin{abstract}
Preattentive texture discrimination is thought to occur as the result of differences in the firstorder statistics of conspicuous local features known as textons (Julesz, 1984a, 1984b). The present study investigated discrimination as a function of two texton properties: (1) blob contrast and (2) texton conspicuity as a function of contrast polarity. The results demonstrated that preattentive texture discrimination is a function of differences in contrast distribution, and that conspicuity varies with texton type.
\end{abstract}

According to the texton theory (Julesz, 1981, 1984a, 1984b), preattentive texture discrimination results from differences in the first-order statistics of conspicuous local features known as textons. Briefly, textons include elongated blobs, the endpoints of very elongated blobs (terminators), and the regions of crossing of very elongated crossed blobs (crossings). Blobs are further defined as being either On or Off, depending on whether contrast polarity is positive or negative (Julesz, 1982). In the present study we sought to investigate the effects of some hitherto unexamined texton properties of texture discriminability: blob contrast and blob and terminator conspicuity as a function of contrast polarity.

\section{THE ROLE OF BLOB CONTRAST}

Blob contrast can hold two levels of importance in preattentive perception. At one level it may be utilized as blob phase (position) is utilized-simply to signal the presence of textons. Alternatively, blob contrast may be extensively utilized to produce discrimination as a function of differences in the distribution of blob contrast levels.

To determine the status of blob contrast, we used four sets of four texture pairs. One set, which differed from the remaining sets only with respect to blob contrast, is presented in Figure 1. For the Different textures, there are two sources of discrimination. The first is the difference in terminator density: each arrow possesses three terminators, whereas the triangles have no terminators (Julesz, 1982). The second is that each triangle, unlike the arrows, contains a large blob formed by the internal perimeter of the triangle (Julesz, 1984a).

The first set of texture pairs (shown in Figure 1) differed from the second set only in that the micropatterns of the latter were stochastically assigned one of three different contrast levels, producing an identical mottled pattern across each texture. The third and fourth sets of

The authors would like to thank David Badcock and two anonymous reviewers for comments and helpful suggestions in preparing this article. Requests for reprints should be addressed to Steven Taylor, Department of Psychology, University of Melbourne, Parkville 3052, Australia.
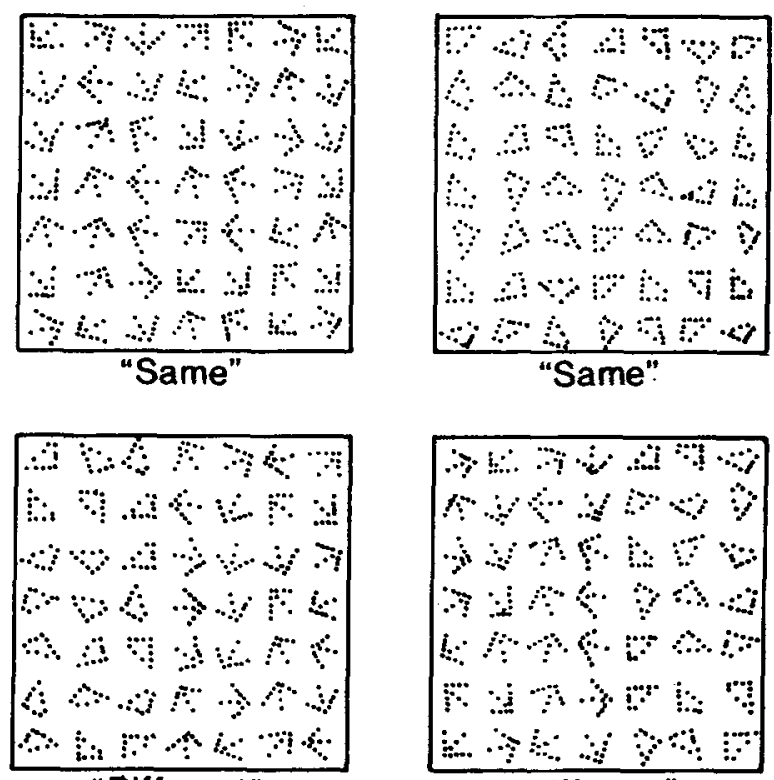

"Different"

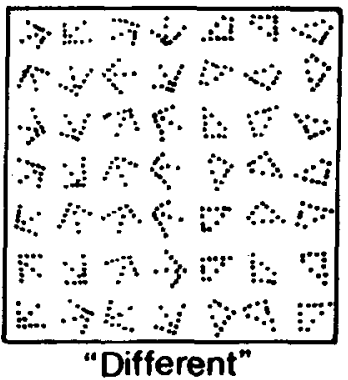

Figure 1. One of the sets of four texture pairs used, with corresponding correct responses.

textures were simply the contrast reversals (negatives) of the first and second sets, respectively. Thus, the four sets of texture pairs were Off Two-tone, Off Mottled, On Twotone, and On Mottled texture pairs.

To examine the effects of blob contrast, we compared the discriminability of Two-tone texture pairs with that of Mottled texture pairs. Although texture discrimination for these textures is not based on blob contrast, we hypothesized that if blob contrast is an important variable in preattentive perception, then the presence of sufficiently salient mottling should function as noise relative to the above-mentioned bases of discriminability (signal). If blob contrast is an important preattentive variable, then an increase in the number of blob contrast levels from one (Two-tone) to three (Mottled) should increase the ratio of signal to noise, consequently affecting discriminability. 
Alternatively, if blob contrast is utilized only to indicate the presence of blobs, no such increase in noise should occur, because the presence of different blob contrast levels would not be detected. Thus, the discriminability of Mottled texture pairs should be no different from that of Two-tone texture pairs.

\section{Method}

Subjects. Forty subjects with normal or corrected-to-normal vision were recruited from the University of Melbourne.

Stimuli and Apparatus. Texture pairs $7 \times 7$ micropatterns in size were generated according to the micropattern (and hence blob) contrast values presented in Table 1. Textures were displayed tachistoscopically and were viewed at a distance of $490 \mathrm{~mm}$. Each micropattern subtended a visual angle of 35 ' of arc across the widest axis.

Procedure. Each texture pair was viewed binocularly for an exposure time of $150 \mathrm{msec}$. Two illumination levels were used, corresponding to $45 \%$ and $100 \%$ of the maximum illumination value of the tachistoscope lamp. Texture luminance values are presented in Table 2.

For each subject, texture pairs were individually presented in two blocks of 100 trials. Each block consisted entirely of either On or Off textures. Within each block the frequency of occurrence of each texture type was matched, and order of presentation was randomized. Half the subjects viewed the block of On texture pairs first, and the remaining subjects viewed Off textures first.

Prior to presentation, a given subject fixated upon a dimly illuminated dot centered in the tachistoscope field. This dot disappeared during texture presentation. An experimental trial consisted of presentation of a texture pair to either the left or right of the fixation point. The side of presentation varied randomly. The eccentricity of presentation was such that the midpoint of the vertical boundary between the two textures was $3^{\circ}$ (visual angle) horizon-

Table 1

Contrast Levels of Micropatterns Relative to Their Respective Surrounds

\begin{tabular}{lc}
\hline Texture Type & Micropattern Contrast* \\
\hline On Two-Tone & .70 \\
On Mottled & $.64, .33, .20$ \\
Off Two-Tone & -.71 \\
Off Mottled & $-.68,-.39,-.14$ \\
\hline
\end{tabular}

*Contrast $=(m-s) /(m+s)$ where $m=$ micropattern luminance and $s$ $=$ surround luminance.

Table 2

Texture Luminance Values (cd/m²; Space Averaged Across Textures)

\begin{tabular}{lcc} 
& \multicolumn{2}{c}{$\begin{array}{c}\text { Ilumination } \\
\text { Level }\end{array}$} \\
\cline { 2 - 3 } Texture Type & Low & High \\
\hline On Two-Tone & 0.5 & 1.0 \\
On Mottled & 0.5 & 1.0 \\
Off Two-Tone & 1.9 & 3.8 \\
Off Mottled & 1.8 & 3.6 \\
\hline
\end{tabular}

Table 3

Percentage of Mottled and Two-Tone Textures Correctly Identified as Same or Different (Combined Across Subjects, Illumination Levels, and Contrast Polarities)

\begin{tabular}{lcc}
\hline & Mottled & Two-Tone \\
\hline Same & 80.73 & 88.55 \\
Different & 74.29 & 64.85 \\
Total & 77.51 & 76.70 \\
\hline
\end{tabular}

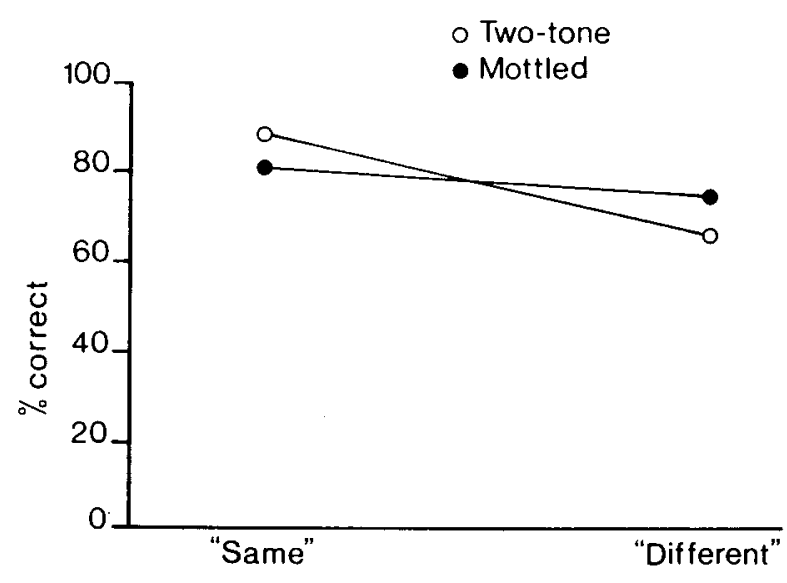

Figure 2. Mottled versus Two-Tone $\times$ Same versus Different interaction.

tally out from the fixation point. Such an arrangement, coupled with the brief presentation time, ensured preattentive viewing conditions (Julesz, 1980). Following presentation, the subject responded "Same" or "Different"; he/she was told to guess if unsure.

\section{Results}

Discriminability was defined by the proportion of texture pairs correctly identified as Same or Different. Discriminability for each texture type is presented in Table 3.

Results were analyzed by means of a four-way ANOVA (On vs. Off $\times$ Mottled vs. Two-tone $\times$ Same vs. Different $\times$ illumination level [high vs. low]). Here, the Mottled vs. Two-tone main effect was found to be nonsignificant $[F(1,38)=0.08, p>.05]$, although the Mottled vs. Two-tone $\times$ Same vs. Different interaction was highly significant $[F(1,38)=47.70, p<.0001]$. For this interaction, simple-effect comparisons revealed that subjects were able to correctly identify Two-tone Same texture pairs significantly more often than Mottled Same texture pairs $[F(1,38)=9.80, p<.01]$, and, conversely, were able to correctly identify Mottled Different texture pairs significantly more often than Two-tone Different texture pairs $[F(1,38)=14.27, p<.005]$. This interaction is displayed in Figure 2.

This result demonstrates that whereas Mottled Different texture pairs were more discriminable than Two-tone Different texture pairs, Mottled Same texture pairs were less discriminable than Two-tone Same texture pairs. Such a finding is inconsistent with the notion that blob contrast is preattentively utilized only to signal the presence of blobs. This effect of mottling was found for On and Off textures alike, as shown by the nonsignificance of the On vs. Off $\times$ Mottled vs. Two-tone interaction $[F(1,38)=$ $3.69, p>.05]$. Also, the effect of illumination level was nonsignificant at the level of main effect $[F(1,38)=0.17$, $p>.05]$ and for all levels of interaction.

In sum, these results demonstrate that blob contrast is an important variable in preattentive texture discrimination. It remains for future research to specify the number and range of blob contrast values that mediate discrimination. 


\section{TEXTON CONSPICUITY AS A FUNCTION OF CONTRAST POLARITY}

Little is known about the relative conspicuity of On blobs, Off blobs, and their respective terminators. Purks and Richards (1977) reported constructing a texture pair for which the Off blob form was more discriminable than its On blob counterpart. Their result, however, arose from discrimination that was not based entirely on preattentive perception, since subjects were permitted to scrutinize textures for several seconds. Furthermore, the difference between their On and Off textures may have been due to differences in the conspicuity of blobs or terminators or both. The aim of the latter part of the present study, therefore, was to investigate the effects of contrast polarity under preattentive conditions in such a way that the effects due to particular textons could be teased out. This was done by performing further analyses of the data presented in the first half of this paper.

Texture discriminability as a function of contrast polarity is presented in Table 4 . Results were analyzed by two ANOVAs: On vs. Off $\times$ Different vs. Same $\times$ illumination level, and On vs. Off $\times$ Same (arrows vs. triangles) $X$ illumination level. For the first ANOVA, the On vs. Off main effect indicated that, overall, On texture pairs were more discriminable than Off texture pairs $[F(1,38)$ $=23.30, p<.0005$ ]. In addition to this, the On vs. Off $\times$ Different vs. Same interaction was nonsignificant $[F(1,38)=3.73, p>.05]$.

For the second ANOVA, the On vs. Off $\times$ Same (arrows vs. triangles) interaction was found to be significant $[F(1,38)=6.86, p<.05]$. A simple-effect comparison indicated that Same texture pairs made up of On arrows were equally as discriminable as those made up of Off arrows $[F(1,38)=0.55, p>.05]$. This shows that the conspicuity of blobs (formed by the strings of collinear dots), as well as that of their terminators, did not change with contrast polarity. This, in turn, reveals that although On micropattern textures were more discriminable overall than Off micropattern textures, the terminators and their corresponding blobs were not the textons responsible for this effect. Therefore, the effect must have been due to the other type of texton that mediated discrimination, namely, the large blobs formed by the internal perimeters of the triangles. This conclusion was confirmed by a subsequent simple-effect comparison made for the On vs. Off $\times$ Same (arrows vs. triangles) interaction. Here, Same texture pairs made up of On triangles were found to be more discriminable than their Off

Table 4

Percentage of On and Off Textures Correctly Identified as Same or Different (Summed Across Subjects, Texture Type [Mottled vs. Two-Tone], and Hlumination Level)

\begin{tabular}{lcc}
\hline & On & Off \\
\hline Same: Arrows & 89.70 & 87.54 \\
Same: Triangles & 85.28 & 75.51 \\
Different & 74.75 & 64.40 \\
Total & 84.24 & 72.97 \\
\hline
\end{tabular}

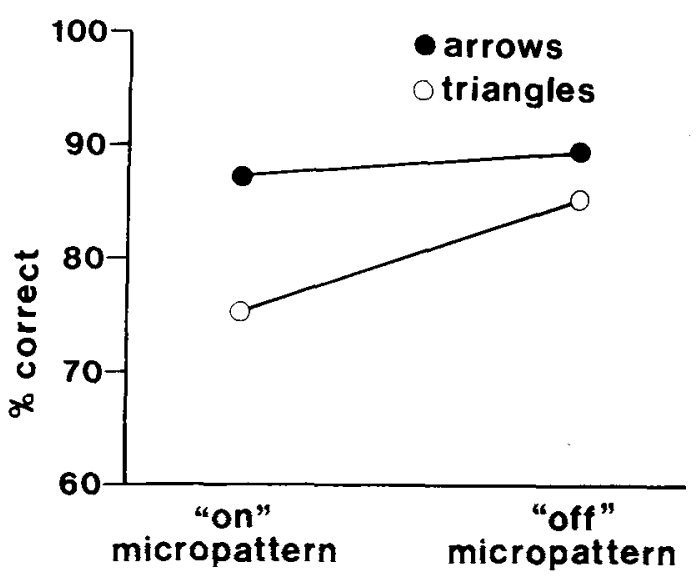

Figure 3. On versus Off $\times$ arrows versus triangles interaction.

counterparts $[F(1,38)=11.31, p<.005]$. This interaction is shown in Figure 3. Thus, it appears that the On micropattern texture pairs were more discriminable than the Off micropattern texture pairs because the large Off blobs formed by each On micropattern were more conspicuous than the large On blobs formed by the Off micropatterns.

\section{CONCLUSIONS}

The present study has served to further specify the types of parameters important in preattentive texture perception. Unlike blob phase, textural properties such as the magnitude and polarity of blob contrast have been shown to be important in discrimination. Moreover, the findings concerning texton conspicuity demonstrate that discriminability is not simply a function of differences in the first-order statistics of textons. A task for subsequent research is to investigate the possibility of an interaction or tradeoff between the detection of conspicuity differences and texton first-order statistic differences.

\section{REFERENCES}

JuLESz, B. (1980). Figure and ground perception in briefly presented isodipole textures. In E. Kubovy \& J. R. Pomerantz (Eds.), Perceptual organization (pp. 27-54). Hillsdale, NJ: Erlbaum.

JuLesz, B. (1981). Textons, the elements of texture perception and their interactions. Nature (London), 290, 91-97.

JuLesz, B. (1982). The role of terminators in preattentive perception of line textures. In D. G. Albrecht (Ed.), Lecture notes in biomathematics: 44. Recognition of pattern and form (pp. 33-58). New York: Springer-Verlag.

JULESZ, B. (1984a). Adaptation in a peephole: A texton theory of preattentive vision. In L. Spillman \& B. R. Wooten (Eds.), Sensory experience, adaptation, and perception: Festschrift for Ivo Kohler (pp. 37-52). Hillsdale, NJ: Erlbaum.

JULESZ, B. (1984b). A brief outline of the texton theory of human vision. Trends in Neurosciences, 2, 41-45.

Purks, S. R., \& Richards, W. (1977). Visual texture discrimination using random-dot patterns. Joumal of the Optical Society of America, 67, 765-771.

(Manuscript received August 30, 1985; revision accepted for publication July 28,1986 .) 